changed as recruits became better nourished and healthier. Modern technology has updated the methodology for data collection, although not necessarily beneficially.

Conclusion Although the military population, its health problems and the methods of data collection have changed over time, the fundamental principle of basing military health protection on sound epidemiology remains constant. The lessons of the past provide evidence on which future planning can be based.

\section{P1-90 SOCIAL INEQUALITIES IN HEALTH AMONG ELDERLY IN A BRAZILIAN SOUTHEASTERN CITY}

doi:10.1136/jech.2011.142976c.83

${ }^{1} \mathrm{M}$ B de Azevedo Barros, ${ }^{*}{ }^{1} \mathrm{P}$ M B Francisco, ${ }^{1} \mathrm{M}$ G Lima, ${ }^{2} \mathrm{C}$ L G Cesar. ${ }^{1}$ State University of Campinas Medical School, Campinas, São Paulo, Brazil, ${ }^{2}$ University of São Paulo, Faculty of Public Health, São Paulo, Brazil

Introduction Social inequalities in health is an important problem in Brazil challenging the public Health System. This is also an issue that affects the elderly population.

Objective The aim of this study was to assess the magnitude of social inequalities in health status, health behaviour and use of health services in elderly.

Methods A population-based cross-sectional study was carried out in 2008 e 2009 involving 1518 elderly residents of Campinas, SP, Brazil (ISACAMP 2008/2009). Social inequalities were assessed by educational level. Prevalence and adjusted prevalence ratios were estimated applying Poisson multiple regression, using svy commands of Stata11.

Results Significant social differences were found between the educational strata. Elderly individuals with a higher degree of schooling consume more alcoholic beverages $(R P=1.94)$, are less sedentary $(\mathrm{RP}=0.72)$, have healthier dietary (1.64) and a lower prevalence of hypertension $(\mathrm{RP}=0.80)$, diabetes $(\mathrm{RP}=0.71)$, dizziness $(\mathrm{RP}=0.67)$, headaches $(\mathrm{RP}=0.52)$, back pain $(\mathrm{RP}=0.77)$, visual impairment $(\mathrm{RP}=0.57)$ and denture use $(\mathrm{RP}=0.68)$. But, there were no differences in the use of health services in the previous 2 weeks, in hospitalisation or in surgeries in the previous year and in medicine intake in the previous 3 days. Among elderly with hypertension or diabetes, there were also no differences in the regular use of health services and medication.

Conclusion The results showed strong social inequality in this elderly population with significant differences in several health indicators, along with equity in the access of some health service components, suggesting positive effects of the Brazilian Public Health System on promoting health equity.

\section{P1-91 PREVALENCE AND TEMPORAL TRENDS IN THE PREVALENCE OF SMALL INTESTINAL ATRESIA IN EUROPE: A MULTILEVEL ANALYSIS}

doi:10.1136/jech.2011.142976c.84

${ }^{1} \mathrm{~K}$ E Best, ${ }^{* 1} \mathrm{P}$ W G Tennant, ${ }^{1,2} \mathrm{~J}$ Rankin, ${ }^{3}$ Eurocat Working Group. ${ }^{1}$ Newcastle University, Newcastle upon Tyne, UK; ${ }^{2}$ Regional Maternity Survey Office, Newcastle upon Tyne, UK; ${ }^{3}$ University of Ulster, Belfast, UK

Introduction Small intestinal atresia (SIA) is a congenital anomaly characterised by the abnormal closure, discontinuity or narrowing of the duodenum, jejunum or ileum. This study used multilevel regression to examine the total prevalence and temporal trends in the prevalence of SIA in Europe.

Methods Cases of SIA delivered during 1990-2006 and notified to 21 European congenital anomaly registers formed this populationbased case series. Total prevalence and changes in prevalence over time were modelled using multilevel Poisson regression. Heterogeneity between registers was evaluated from the intercept's random component. Inter-regional differences in trends were examined by including random slopes.

Results 1154 SIA cases were reported among 5383099 registered births. Of 1092 singleton cases, 222 (20.3\%) were associated with chromosomal and 227 (20.8\%) with structural anomalies. The prevalence per 10000 births for singleton cases of normal karyotype was 1.6 (95\% CI 1.5 to 1.7$)$ for SIA, 0.9 (95\% CI 0.8 to 1.0$)$ for duodenal atresia and 0.8 (95\% CI 0.7 to 0.8 ) for JIA. There was no significant trend in SIA, duodenal atresia or JIA prevalence over time $(\mathrm{RR}=1.0,95 \%$ credible interval (CrI): 1.0 to 1.0 , for each) but SIA and duodenal atresia prevalence varied significantly between participating registers ( $p=0.03$ and $p=0.04$, respectively). There was no increased risk of SIA in mothers aged $<20$ years compared to mothers aged 20 to 29 ( $R R=1.3,95 \%$ CrI: 1.0 to $1.8 ; p=0.08$ ).

Conclusion This study found no evidence of a temporal trend in the prevalence of SIA, duodenal atresia or JIA although SIA and duodenal atresia rates varied between geographic areas.

\section{P1-92 AN EPIDEMIOLOGICAL STUDY OF TUBERCULOSIS PATIENTS WITH RISK PATTERN OF HIV/AIDS AMONG UNDERPRIVILEGED POPULATION IN NORTH INDIA}

doi:10.1136/jech.2011.142976c.85

V Bhatia, ${ }^{*}$ S Puri, M Thakare. Government Medical College, Sector 32, Chandigarh, India

Introduction Tuberculosis is a major cause of morbidity and mortality in developing countries. National AIDS Control Organization, India has reported $\mathrm{TB}$ as the commonest opportunistic infection (62.3\%) among HIV infected persons.

Methodology The study was conducted among 155 patients of tuberculosis at three Microcopy centres attending urban and rural health centres with the objectives of studying epidemiological profile of patients and to assess HIV-AIDS pattern and high risk behaviour. Information on pres-designed format related to socio-demographic clinical profile, categorisation, treatment and awareness about HIVAIDS, mode of transmission and behaviour was gathered in 2009.

Results Maximum number of patients were in the age group of $21-30$ yrs (23.22\%). $41.93 \%$ being illiterate. $42.58 \%$ were having a monthly family income of under Rs. 3000/- (US\$ 67). $47.74 \%$ had migrated from another poor state, $70.32 \%$ married. $11.72 \%$ TB patients were staying with under-six children. Fever $(79.35 \%)$ and cough $(72.25 \%)$ were presenting symptoms at starting the treatment. $54.19 \%$ belonged to category I of DOTS. Only half (54.19\%) were aware of HIV-AIDS- $77.35 \%$ in rural and $42.15 \%$ in urban areas. History of multiple partners could be elicited from two cases in urban settings History of blood transfusion was given by 7 (4.51\%), 18 (11.61\%) of TB were tested for HIV. $77.35 \%$ from rural and $23.52 \%$ from urban areas (total 21.93\%) desired to know their HIV status.

Conclusion Maximum numbers of TB cases were in young agegroup, males, low socio-economic status particularly in urban slums with poor awareness level about HIV/AIDS. Coordinated efforts for implementation of the two programs for such population groups are required in controlling these diseases.

\section{P1-93 TRENDS IN THE BURDEN OF CARDIOVASCULAR DISEASES IN THE UK, 1961 TO 2011}

doi:10.1136/jech.2011.142976c.86

P Bhatnagar, ${ }^{*}$ P Scarborough, K Wickramasinghe. University of Oxford, Oxford, UK

Introduction Mortality from cardiovascular disease (CVD) has dramatically reduced over the past 50 years in the UK. While this trend should be celebrated, it is important to consider mortality alongside trends in morbidity to gain a full understanding of how healthcare resources and prevention schemes should be directed. 
This paper reported trends in mortality, incidence and prevalence of coronary heart disease (CHD), stroke, heart attack, angina and heart failure over the past 50 years.

Methods Mortality data were provided by the UK national statistics agencies. For morbidity data we reviewed the peer-reviewed and grey literature for comparable estimates from different time points over the last 50 years.

Results Around half of the UK population died from CVD in the 1960s; by 2009 this had dropped to a third. CHD mortality rates have remained 30\%-40\% higher in Scotland than in England since 1961. Incidence rates for heart attack have decreased since the 1960s, while survival has improved; prevalence in those over 75 has increased by around $40 \%$ since the mid-1990s. Over the past 20 years heart failure incidence decreased by over a third in Scotland. Between 1970 and 1991, prevalence of angina nearly tripled for men over 75 and has continued to rise.

Conclusion Mortality from CVD has declined over the past 50 years, but striking geographic inequalities have remained. Incidence of two major CVD conditions have declined, but continuing increases in prevalence and an ageing population mean that the burden of CVD is still a major issue for the UK.

\section{P1-94 THE EPIDEMIOLOGY AND COSTS OF ANKLE INJURIES: A REVIEW OF THE LITERATURE}

doi:10.1136/jech.2011.142976c.87

I A Bielska, * A Johnson. Queen's University, Kingston, Ontario, Canada

Introduction Ankle sprains are one of the most common injuries presenting to emergency departments, representing $3 \%$ to $5 \%$ of all visits in the UK, and $10 \%$ of all injury-related visits in the USA. Ankle injuries have significant physical and economic consequences for the affected individuals.

Objectives To describe the epidemiology of ankle sprains and fractures among the general population; and to determine the direct and indirect costs related to the diagnosis and treatment of ankle injuries.

Methods A comprehensive literature review of Ovid MEDLINE, EMBASE, Cochrane DSR, ACP Journal Club, AMED, Ovid Healthstar, and CINAHL was conducted for English-language studies on ankle sprains and fractures published from 1980 to 2010.

Results The search identified 2394 studies of which 47 were selected for analysis. A majority of the studies were published in the last decade. The incidence of ankle sprains was 2 to 7 per 1000 personyears, while the incidence of ankle fractures was 1 per 1000 personyears. The costs of emergency ankle sprain management ranged from 126.13 to 2356.21 per patient (2009 CAD), depending on sprain severity. The management costs were higher for ankle fractures: 1692.82 to 15802.26 (2009 CAD) per patient. The economic evaluations were conducted from the societal or healthcare system perspective.

Conclusions Information on the epidemiology of ankle sprains and fractures may help plan for health policy and the provision of health services. Moreover, the cost data may inform future studies undertaking economic evaluations of the diagnosis and treatment of ankle injuries.

\section{P1-95 IS CURRENT POLICY FOR ANAEMIA PREVENTION IN BEDOUIN TODDLERS IN THE NEGEV APPROPRIATE?}

doi:10.1136/jech.2011.142976c.88

\footnotetext{
${ }^{1,2} \mathrm{~N}$ Bilenko, ${ }^{* 1,3} \mathrm{D}$ Fraser, ${ }^{4} \mathrm{M}$ Tatikashvili, ${ }^{2,3}$ I Belmaker. ${ }^{1}$ Department of Epidemiology and Health Services Evaluation, Ben-Gurion University of the Negev, Beer-Sheva, Israel; ${ }^{2}$ Regional Office of Ministry of Health, Southern Region, Beer-Sheva, Israel; ${ }^{3}$ S. Daniel Abraham International Center for Health and Nutrition, Ben-Gurion University of
}

the Negev, Beer-Sheva, Israel; ${ }^{4}$ Soroka University Medical Center, Beer-Sheva, Israel; ${ }^{5}$ Community Health Division, Ben-Gurion University of the Negev, Beer-Sheva, Israel

Introduction Iron deficiency anaemia still affects a quarter of the world's population increasing risk of infectious disease morbidity, impaired growth and mental development. According to current policy children undergo screening for iron deficiency anaemia at age 9-12M. Methods Prospective Study The study population included Moslem Bedouin 2.5-3-Y-old children that followed from 6M in Well Baby Clinic. All parents of participants were interviewed during enrolment and monthly meetings. The blood samples were taken from children at enrolment and during last follow-up visit. Anaemia $(\mathrm{Hb}<11 \mathrm{~g} / \mathrm{dl})$ and Iron Deficiency Index (at least 2 of 6 abnormal indicators, including Haemoglobin, Haematocrit, Mean Corpuscular Volume, Red blood cell distribution Width, serum ferritin, and transferin saturation) were defined.

Results The study population included 180 infants. The mother's young age was found as a risk factor for mild anaemia. Male sex was associated with a higher rate of moderate anaemia compared with female sex $(76.2 \%$ and $52.8 \%$, respectively, $p=0.043)$. Children with anaemia had lower average of dietary iron consumption than children with normal levels of $\mathrm{Hb}(\mathrm{p}=0.009)$. Iron deficiency anaemia at age $6 \mathrm{M}$ was a significant and independent risk factor for toddlers' anaemia $(\mathrm{OR}=3.47, \mathrm{p}<0.001)$ controlling for the mother's age, child gender and consumption of dietary iron

Conclusion The most significant factor for anaemia among this population is iron deficiency anaemia at the age of $6 \mathrm{M}$. Prevention, early detection (at age 6M) and appropriate treatment of anaemia in the first year of life are critical to prevent anaemia and its consequences later life.

\section{P1-96 PRIMARY BONE CANCER IN 0-49 YEAR OLDS IN GREAT BRITAIN, 1980-2005 AND FLUORIDE IN DRINKING WATER: A CASE OF INEQUALITIES?}

doi:10.1136/Jech.2011.142976c.89

${ }^{1} \mathrm{~K}$ Blakey, ${ }^{*} \mathrm{R}$ Feltbower, ${ }^{2} \mathrm{R}$ Parslow, ${ }^{1} \mathrm{P}$ James, ${ }^{1} \mathrm{~B}$ G Pozo, ${ }^{3} \mathrm{C}$ Stiller, ${ }^{3} \mathrm{~T}$ Vincent, ${ }^{3} \mathrm{P}$ Norman, ${ }^{2} \mathrm{P}$ McKinney, ${ }^{3} \mathrm{M}$ Murphy, ${ }^{5} \mathrm{~A}$ Craft, ${ }^{1} \mathrm{R}$ McNally. ${ }^{1}$ Institute of Health 8 Society, Newcastle University, Newcastle-upon-Tyne, UK; ${ }^{2}$ Paediatric Epidemiology Group, University of Leeds, Leeds, UK; ${ }^{3}$ Childhood Cancer Research Group, Department of Paediatrics, University of Oxford, Oxford, UK; ${ }^{4}$ School of Geography, University of Leeds, Leeds, UK; ${ }^{5}$ Northern Institute of Cancer Research, Newcastle University, Newcastle-upon-Tyne, UK

Introduction Primary bone cancers (PBC) occur most often in young people. Osteosarcoma and Ewing sarcoma family of bone tumours (ESFT) are most commonly diagnosed in children but aetiology remains unclear. Fluoride has been proposed as a potential causal agent for $\mathrm{PBC}$. The study investigated whether incidence of PBC was linked with fluoride in drinking water.

Method Incidence data on cases aged $<50$ years diagnosed during 1980-2005 were obtained from all ten regional cancer registries in Great Britain (GB). These data were combined with small-area population census, digital boundary and fluoride monitoring data. Negative binomial regression was used to examine the relationship between incidence rates and census small-area fluoride levels. These models were fitted to small-area census data aggregated into three age bands and by gender with the logarithm of the 'at risk' population as an offset.

Results There were 2566 osteosarcoma cases aged 0-49 years: 817 aged $0-14 ; 1315$ aged $15-29$ and 434 aged $30-49$ years. For ESFT there were 1650 cases aged $0-49$ years: 659 aged $0-14 ; 800$ aged 15-29 and 191 aged 30-49 years. After adjustment for age and gender, no statistically significant association was found between osteosarcoma or ESFT and fluoride: RR for one part per million increase in fluoride level $=0.993$; $95 \%$ CI 0.843 to 1.171 and 0.860 ; 95\% CI 0.696 to 1.064 respectively. 